\title{
COMPLETE INTERSECTION MONOMIAL CURVES AND THE COHEN-MACAULAYNESS OF THEIR TANGENT CONES
}

\author{
ANARGYROS KATSABEKIS
}

\begin{abstract}
Let $C(\mathbf{n})$ be a complete intersection monomial curve in the 4dimensional affine space. In this paper we study the complete intersection property of the monomial curve $C(\mathbf{n}+w \mathbf{v})$, where $w>0$ is an integer and $\mathbf{v} \in \mathbb{N}^{4}$. Also we investigate the Cohen-Macaulayness of the tangent cone of $C(\mathbf{n}+w \mathbf{v})$.
\end{abstract}

\section{INTRODUCTION}

Let $\mathbf{n}=\left(n_{1}, n_{2}, \ldots, n_{d}\right)$ be a sequence of positive integers with $\operatorname{gcd}\left(n_{1}, \ldots, n_{d}\right)=$ 1. Consider the polynomial ring $K\left[x_{1}, \ldots, x_{d}\right]$ in $d$ variables over a field $K$. We shall denote by $\mathbf{x}^{\mathbf{u}}$ the monomial $x_{1}^{u_{1}} \cdots x_{d}^{u_{d}}$ of $K\left[x_{1}, \ldots, x_{d}\right]$, with $\mathbf{u}=\left(u_{1}, \ldots, u_{d}\right) \in \mathbb{N}^{d}$ where $\mathbb{N}$ stands for the set of non-negative integers. The toric ideal $I(\mathbf{n})$ is the kernel of the $K$-algebra homomorphism $\phi: K\left[x_{1}, \ldots, x_{d}\right] \rightarrow K[t]$ given by

$$
\phi\left(x_{i}\right)=t^{n_{i}} \text { for all } 1 \leq i \leq d .
$$

Then $I(\mathbf{n})$ is the defining ideal of the monomial curve $C(\mathbf{n})$ given by the parametrization $x_{1}=t^{n_{1}}, \ldots, x_{d}=t^{n_{d}}$. The ideal $I(\mathbf{n})$ is generated by all the binomials $\mathbf{x}^{\mathbf{u}}-\mathbf{x}^{\mathbf{v}}$, where $\mathbf{u}-\mathbf{v}$ runs over all vectors in the lattice $\operatorname{ker}_{\mathbb{Z}}\left(n_{1}, \ldots, n_{d}\right)$ see for example, [16, Lemma 4.1]. The height of $I(\mathbf{n})$ is $d-1$ and also equals the rank of $\operatorname{ker}_{\mathbb{Z}}\left(n_{1}, \ldots, n_{d}\right)$ (see [16]). Given a polynomial $f \in I(\mathbf{n})$, we let $f_{*}$ be the homogeneous summand of $f$ of least degree. We shall denote by $I(\mathbf{n})_{*}$ the ideal in $K\left[x_{1}, \ldots, x_{d}\right]$ generated by the polynomials $f_{*}$ for $f \in I(\mathbf{n})$.

Deciding whether the associated graded ring of the local ring $K\left[\left[t^{n_{1}}, \ldots, t^{n_{d}}\right]\right]$ is Cohen-Macaulay constitutes an important problem studied by many authors, see for instance [1, 6], 14. The importance of this problem stems partially from the fact that if the associated graded ring is Cohen-Macaulay, then the Hilbert function of $K\left[\left[t^{n_{1}}, \ldots, t^{n_{d}}\right]\right]$ is non-decreasing. Since the associated graded ring of $K\left[\left[t^{n_{1}}, \ldots, t^{n_{d}}\right]\right]$ is isomorphic to the ring $K\left[x_{1}, \ldots, x_{d}\right] / I(\mathbf{n})_{*}$, the CohenMacaulayness of the associated graded ring can be studied as the Cohen-Macaulayness of the ring $K\left[x_{1}, \ldots, x_{d}\right] / I(\mathbf{n})_{*}$. Recall that $I(\mathbf{n})_{*}$ is the defining ideal of the tangent cone of $C(\mathbf{n})$ at 0 .

The case that $K\left[\left[t^{n_{1}}, \ldots, t^{n_{d}}\right]\right]$ is Gorenstein has been particularly studied. This is partly due to the M. Rossi's problem [13] asking whether the Hilbert function of a Gorenstein local ring of dimension one is non-decreasing. Recently, A. Oneto, F. Strazzanti and G. Tamone [12] found many families of monomial curves giving negative answer to the above problem. However M. Rossi's problem is still open for a Gorenstein local ring $K\left[\left[t^{n_{1}}, \ldots, t^{n_{4}}\right]\right]$. It is worth to note that, for a complete intersection monomial curve $C(\mathbf{n})$ in the 4-dimensional affine space (i.e. the ideal $I(\mathbf{n})$ is a complete intersection), we have, from [14, Theorem 3.1], that if the minimal number of generators for $I(\mathbf{n})_{*}$ is either three or four, then $C(\mathbf{n})$ has

2010 Mathematics Subject Classification. 14M10, 14M25, 13H10.

Key words and phrases. Monomial curve, Complete intersection, Tangent cone. 
Cohen-Macaulay tangent cone at the origin. The converse is not true in general, see [14, Proposition 3.14].

In recent years there has been a surge of interest in studying properties of the monomial curve $C(\mathbf{n}+w \mathbf{v})$, where $w>0$ is an integer and $\mathbf{v} \in \mathbb{N}^{d}$, see for instance [4, [7] and [18. This is particularly true for the case that $\mathbf{v}=(1, \ldots, 1)$. In fact, J. Herzog and H. Srinivasan conjectured that if $n_{1}<n_{2}<\cdots<n_{d}$ are positive numbers, then the Betti numbers of $I(\mathbf{n}+w \mathbf{v})$ are eventually periodic in $w$ with period $n_{d}-n_{1}$. The conjecture was proved by $\mathrm{T}$. Vu [18]. More precisely, he showed that there exists a positive integer $N$ such that, for all $w>N$, the Betti numbers of $I(\mathbf{n}+w \mathbf{v})$ are periodic in $w$ with period $n_{d}-n_{1}$. The bound $N$ depends on the Castelnuovo-Mumford regularity of the ideal generated by the homogeneous elements in $I(\mathbf{n})$. For $w>\left(n_{d}-n_{1}\right)^{2}-n_{1}$ the minimal number of generators for $I(\mathbf{n}+w(1, \ldots, 1))$ is periodic in $w$ with period $n_{d}-n_{1}$ (see [4]). Furthermore, for every $w>\left(n_{d}-n_{1}\right)^{2}-n_{1}$ the monomial curve $C(\mathbf{n}+w(1, \ldots, 1))$ has Cohen-Macaulay tangent cone at the origin, see [15]. The next example provides a monomial curve $C(\mathbf{n}+w(1, \ldots, 1))$ which is not a complete intersection for every $w>0$.

Example 1.1. Let $\mathbf{n}=(15,25,24,16)$, then $I(\mathbf{n})$ is a complete intersection on the binomials $x_{1}^{5}-x_{2}^{3}, x_{3}^{2}-x_{4}^{3}$ and $x_{1} x_{2}-x_{3} x_{4}$. Consider the vector $\mathbf{v}=(1,1,1,1)$. For every $w>85$ the minimal number of generators for $I(\mathbf{n}+w \mathbf{v})$ is either 18,19 or 20. Using CoCoA ([3]) we find that for every $0<w \leq 85$ the minimal number of generators for $I(\mathbf{n}+w \mathbf{v})$ is greater than or equal to 4 . Thus for every $w>0$ the ideal $I(\mathbf{n}+w \mathbf{v})$ is not a complete intersection.

Given a complete intersection monomial curve $C(\mathbf{n})$ in the 4-dimensional affine space, we study (see Theorems 2.6, 3.2) when $C(\mathbf{n}+w \mathbf{v}$ ) is a complete intersection. We also construct (see Theorems 2.8, 2.9. 3.4) families of complete intersection monomial curves $C(\mathbf{n}+w \mathbf{v})$ with Cohen-Macaulay tangent cone at the origin.

Let $a_{i}$ be the least positive integer such that $a_{i} n_{i} \in \sum_{j \neq i} \mathbb{N} n_{j}$. To study the complete intersection property of $C(\mathbf{n}+w \mathbf{v})$ we use the fact that after permuting variables, if necessary, there exists (see [14, Proposition 3.2] and also Theorems 3.6 and 3.10 in [10]) a minimal system of binomial generators $S$ of $I(\mathbf{n})$ of the following form:

(A) $S=\left\{x_{1}^{a_{1}}-x_{2}^{a_{2}}, x_{3}^{a_{3}}-x_{4}^{a_{4}}, x_{1}^{u_{1}} x_{2}^{u_{2}}-x_{3}^{u_{3}} x_{4}^{u_{4}}\right\}$.

(B) $S=\left\{x_{1}^{a_{1}}-x_{2}^{a_{2}}, x_{3}^{a_{3}}-x_{1}^{u_{1}} x_{2}^{u_{2}}, x_{4}^{a_{4}}-x_{1}^{v_{1}} x_{2}^{v_{2}} x_{3}^{v_{3}}\right\}$.

In section 2 we focus on case (A). We prove that the monomial curve $C(\mathbf{n})$ has Cohen-Macaulay tangent cone at the origin if and only if the minimal number of generators for $I(\mathbf{n})_{*}$ is either three or four. Also we explicitly construct vectors $\mathbf{v}_{i}, 1 \leq i \leq 22$, such that for every $w>0$, the ideal $I\left(\mathbf{n}+w \mathbf{v}_{i}\right)$ is a complete intersection whenever the entries of $\mathbf{n}+w \mathbf{v}_{i}$ are relatively prime. We show that if $C(\mathbf{n})$ has Cohen-Macaulay tangent cone at the origin, then for every $w>0$ the monomial curve $C\left(\mathbf{n}+w \mathbf{v}_{1}\right)$ has Cohen-Macaulay tangent cone at the origin whenever the entries of $\mathbf{n}+w \mathbf{v}_{1}$ are relatively prime. Additionally we show that there exists a non-negative integer $w_{0}$ such that for all $w \geq w_{0}$, the monomial curves $C\left(\mathbf{n}+w \mathbf{v}_{9}\right)$ and $C\left(\mathbf{n}+w \mathbf{v}_{13}\right)$ have Cohen-Macaulay tangent cones at the origin whenever the entries of the corresponding sequence $\left(\mathbf{n}+w \mathbf{v}_{9}\right.$ for the first family and $\mathbf{n}+w \mathbf{v}_{13}$ for the second) are relatively prime. Finally we provide an infinite family of complete intersection monomial curves $C_{m}\left(\mathbf{n}+w \mathbf{v}_{1}\right)$ with corresponding local rings having non-decreasing Hilbert functions, although their tangent cones are not Cohen-Macaulay, thus giving a positive partial answer to M. Rossi's problem.

In section 3 we study the case $(\mathrm{B})$. We construct vectors $\mathbf{b}_{i}, 1 \leq i \leq 22$, such that for every $w>0$, the ideal $I\left(\mathbf{n}+w \mathbf{b}_{i}\right)$ is a complete intersection whenever 
the entries of $\mathbf{n}+w \mathbf{b}_{i}$ are relatively prime. Furthermore we show that there exists a non-negative integer $w_{1}$ such that for all $w \geq w_{1}$, the ideal $I\left(\mathbf{n}+w \mathbf{b}_{22}\right)_{*}$ is a complete intersection whenever the entries of $\mathbf{n}+w \mathbf{b}_{22}$ are relatively prime.

\section{The CaSe (A)}

In this section we assume that after permuting variables, if necessary, $S=\left\{x_{1}^{a_{1}}-\right.$ $\left.x_{2}^{a_{2}}, x_{3}^{a_{3}}-x_{4}^{a_{4}}, x_{1}^{u_{1}} x_{2}^{u_{2}}-x_{3}^{u_{3}} x_{4}^{u_{4}}\right\}$ is a minimal generating set of $I(\mathbf{n})$. First we will show that the converse of [14, Theorem 3.1] is also true in this case.

Let $n_{1}=\min \left\{n_{1}, \ldots, n_{4}\right\}$ and also $a_{3}<a_{4}$. By [6, Theorem 7] a monomial curve $C(\mathbf{n})$ has Cohen-Macaulay tangent cone if and only if $x_{1}$ is not a zero divisor in the ring $K\left[x_{1}, \ldots, x_{4}\right] / I(\mathbf{n})_{*}$. Hence if $C(\mathbf{n})$ has Cohen-Macaulay tangent cone at the origin, then $I(\mathbf{n})_{*}:\left\langle x_{1}\right\rangle=I(\mathbf{n})_{*}$. Without loss of generality we can assume that $u_{2} \leq a_{2}$. In case that $u_{2}>a_{2}$ we can write $u_{2}=g a_{2}+h$, where $0 \leq h<a_{2}$. Then we can replace the binomial $x_{1}^{u_{1}} x_{2}^{u_{2}}-x_{3}^{u_{3}} x_{4}^{u_{4}}$ in $S$ with the binomial $x_{1}^{u_{1}+g a_{1}} x_{2}^{h}-x_{3}^{u_{3}} x_{4}^{u_{4}}$. Without loss of generality we can also assume that $u_{3} \leq a_{3}$.

Theorem 2.1. Suppose that $u_{3}>0$ and $u_{4}>0$. Then $C(\mathbf{n})$ has Cohen-Macaulay tangent cone at the origin if and only if the ideal $I(\mathbf{n})_{*}$ is either a complete intersection or an almost complete intersection.

Proof. $(\Longleftarrow)$ If the minimal number of generators of $I(\mathbf{n})_{*}$ is either three or four, then $C(\mathbf{n})$ has Cohen-Macaulay tangent cone at the origin.

$(\Longrightarrow)$ Let $f_{1}=x_{1}^{a_{1}}-x_{2}^{a_{2}}, f_{2}=x_{3}^{a_{3}}-x_{4}^{a_{4}}, f_{3}=x_{1}^{u_{1}} x_{2}^{u_{2}}-x_{3}^{u_{3}} x_{4}^{u_{4}}$. We distinguish the following cases

(1) $u_{2}<a_{2}$. Note that $x_{4}^{a_{4}+u_{4}}-x_{1}^{u_{1}} x_{2}^{u_{2}} x_{3}^{a_{3}-u_{3}} \in I(\mathbf{n})$. We will show that $a_{4}+u_{4} \leq u_{1}+u_{2}+a_{3}-u_{3}$. Suppose that $u_{1}+u_{2}+a_{3}-u_{3}<a_{4}+u_{4}$, then $x_{2}^{u_{2}} x_{3}^{a_{3}-u_{3}} \in I(\mathbf{n})_{*}:\left\langle x_{1}\right\rangle$ and therefore $x_{2}^{u_{2}} x_{3}^{a_{3}-u_{3}} \in I(\mathbf{n})_{*}$. Since $\left\{f_{1}, f_{2}, f_{3}\right\}$ is a generating set of $I(\mathbf{n})$, the monomial $x_{2}^{u_{2}} x_{3}^{a_{3}-u_{3}}$ is divided by at least one of the monomials $x_{2}^{a_{2}}$ and $x_{3}^{a_{3}}$. But $u_{2}<a_{2}$ and $a_{3}-u_{3}<a_{3}$, so $a_{4}+u_{4} \leq u_{1}+u_{2}+a_{3}-u_{3}$. Let $G=\left\{f_{1}, f_{2}, f_{3}, f_{4}=\right.$ $\left.x_{4}^{a_{4}+u_{4}}-x_{1}^{u_{1}} x_{2}^{u_{2}} x_{3}^{a_{3}-u_{3}}\right\}$. We will prove that $G$ is a standard basis for $I(\mathbf{n})$ with respect to the negative degree reverse lexicographical order with $x_{3}>x_{4}>x_{2}>x_{1}$. Note that $u_{3}+u_{4}<u_{1}+u_{2}$, since $u_{3}+u_{4} \leq$ $u_{1}+u_{2}+a_{3}-a_{4}$ and also $a_{3}-a_{4}<0$. Thus $\operatorname{LM}\left(f_{3}\right)=x_{3}^{u_{3}} x_{4}^{u_{4}}$. Furthermore $\operatorname{LM}\left(f_{1}\right)=x_{2}^{a_{2}}, \operatorname{LM}\left(f_{2}\right)=x_{3}^{a_{3}}$ and $\operatorname{LM}\left(f_{4}\right)=x_{4}^{a_{4}+u_{4}}$. Therefore $\operatorname{NF}\left(\operatorname{spoly}\left(f_{i}, f_{j}\right) \mid G\right)=0$ as $\operatorname{LM}\left(f_{i}\right)$ and $\operatorname{LM}\left(f_{j}\right)$ are relatively prime, for $(i, j) \in\{(1,2),(1,3),(1,4),(2,4)\}$. We compute $\operatorname{spoly}\left(f_{2}, f_{3}\right)=-f_{4}$, so $\operatorname{NF}\left(\operatorname{spoly}\left(f_{2}, f_{3}\right) \mid G\right)=0$. Next we compute $\operatorname{spoly}\left(f_{3}, f_{4}\right)=x_{1}^{u_{1}} x_{2}^{u_{2}} x_{3}^{a_{3}}-$ $x_{1}^{u_{1}} x_{2}^{u_{2}} x_{4}^{a_{4}}$. Then $\operatorname{LM}\left(\operatorname{spoly}\left(f_{3}, f_{4}\right)\right)=x_{1}^{u_{1}} x_{2}^{u_{2}} x_{3}^{a_{3}}$ and only $\operatorname{LM}\left(f_{2}\right)$ divides $\operatorname{LM}\left(\operatorname{spoly}\left(f_{3}, f_{4}\right)\right)$. Also ecart $\left(\operatorname{spoly}\left(f_{3}, f_{4}\right)\right)=a_{4}-a_{3}=\operatorname{ecart}\left(f_{2}\right)$. Then $\operatorname{spoly}\left(f_{2}, \operatorname{spoly}\left(f_{3}, f_{4}\right)\right)=0$ and $\operatorname{NF}\left(\operatorname{spoly}\left(f_{3}, f_{4}\right) \mid G\right)=0$. By [8. Lemma 5.5.11] $I(\mathbf{n})_{*}$ is generated by the least homogeneous summands of the elements in the standard basis $G$. Thus the minimal number of generators for $I(\mathbf{n})_{*}$ is least than or equal to 4 .

(2) $u_{2}=a_{2}$. Note that $x_{4}^{a_{4}+u_{4}}-x_{1}^{u_{1}+a_{1}} x_{3}^{a_{3}-u_{3}} \in I(\mathbf{n})$. We will show that $a_{4}+u_{4} \leq u_{1}+a_{1}+a_{3}-u_{3}$. Clearly the above inequality is true when $u_{3}=a_{3}$. Suppose that $u_{3}<a_{3}$ and $u_{1}+a_{1}+a_{3}-u_{3}<a_{4}+u_{4}$, then $x_{3}^{a_{3}-u_{3}} \in I(\mathbf{n})_{*}:\left\langle x_{1}\right\rangle$ and therefore $x_{3}^{a_{3}-u_{3}} \in I(\mathbf{n})_{*}$. Thus $x_{3}^{a_{3}-u_{3}}$ is divided by $x_{3}^{a_{3}}$, a contradiction. Consequently $a_{4}+u_{4} \leq u_{1}+a_{1}+a_{3}-u_{3}$. We will prove that $H=\left\{f_{1}, f_{2}, f_{5}=x_{1}^{u_{1}+a_{1}}-x_{3}^{u_{3}} x_{4}^{u_{4}}, f_{6}=x_{4}^{a_{4}+u_{4}}-\right.$ $\left.x_{1}^{u_{1}+a_{1}} x_{3}^{a_{3}-u_{3}}\right\}$ is a standard basis for $I(\mathbf{n})$ with respect to the negative degree reverse lexicographical order with $x_{3}>x_{4}>x_{2}>x_{1}$. Here $\operatorname{LM}\left(f_{1}\right)=$ $x_{2}^{a_{2}}, \operatorname{LM}\left(f_{2}\right)=x_{3}^{a_{3}}, \operatorname{LM}\left(f_{5}\right)=x_{3}^{u_{3}} x_{4}^{u_{4}}$ and $\operatorname{LM}\left(f_{6}\right)=x_{4}^{u_{4}+a_{4}}$. Therefore 
$\operatorname{NF}\left(\operatorname{spoly}\left(f_{i}, f_{j}\right) \mid H\right)=0$ as $\operatorname{LM}\left(f_{i}\right)$ and $\operatorname{LM}\left(f_{j}\right)$ are relatively prime, for $(i, j) \in\{(1,2),(1,5),(1,6),(2,6)\}$. We compute $\operatorname{spoly}\left(f_{2}, f_{5}\right)=-f_{6}$, therefore $\operatorname{NF}\left(\operatorname{spoly}\left(f_{2}, f_{5}\right) \mid H\right)=0$. Furthermore $\operatorname{spoly}\left(f_{5}, f_{6}\right)=x_{1}^{u_{1}+a_{1}} x_{3}^{a_{3}}-$ $x_{1}^{u_{1}+a_{1}} x_{4}^{a_{4}}$ and also $\operatorname{LM}\left(\operatorname{spoly}\left(f_{5}, f_{6}\right)\right)=x_{1}^{u_{1}+a_{1}} x_{3}^{a_{3}}$. Only $\operatorname{LM}\left(f_{2}\right)$ divides $\operatorname{LM}\left(\operatorname{spoly}\left(f_{5}, f_{6}\right)\right)$ and ecart $\left(\operatorname{spoly}\left(f_{5}, f_{6}\right)\right)=a_{4}-a_{3}=\operatorname{ecart}\left(f_{2}\right)$. Then $\operatorname{spoly}\left(f_{2}, \operatorname{spoly}\left(f_{5}, f_{6}\right)\right)=0$ and therefore $\operatorname{NF}\left(\operatorname{spoly}\left(f_{5}, f_{6}\right) \mid H\right)=0$. By 8 , Lemma 5.5.11] $I(\mathbf{n})_{*}$ is generated by the least homogeneous summands of the elements in the standard basis $H$. Thus the minimal number of generators for $I(\mathbf{n})_{*}$ is least than or equal to 4 .

Corollary 2.2. Suppose that $u_{3}>0$ and $u_{4}>0$.

(1) Assume that $u_{2}<a_{2}$. Then $C(\mathbf{n})$ has Cohen-Macaulay tangent cone at the origin if and only if $a_{4}+u_{4} \leq u_{1}+u_{2}+a_{3}-u_{3}$.

(2) Assume that $u_{2}=a_{2}$. Then $\bar{C}(\mathbf{n})$ has Cohen-Macaulay tangent cone at the origin if and only if $a_{4}+u_{4} \leq u_{1}+a_{1}+a_{3}-u_{3}$.

Theorem 2.3. Suppose that either $u_{3}=0$ or $u_{4}=0$. Then $C(\mathbf{n})$ has CohenMacaulay tangent cone at the origin if and only if the ideal $I(\mathbf{n})_{*}$ is a complete intersection.

Proof. It is enough to show that if $C(\mathbf{n})$ has Cohen-Macaulay tangent cone at the origin, then the ideal $I(\mathbf{n})_{*}$ is a complete intersection. Suppose first that $u_{3}=0$. Then $\left\{f_{1}=x_{1}^{a_{1}}-x_{2}^{a_{2}}, f_{2}=x_{3}^{a_{3}}-x_{4}^{a_{4}}, f_{3}=x_{4}^{u_{4}}-x_{1}^{u_{1}} x_{2}^{u_{2}}\right\}$ is a minimal generating set of $I(\mathbf{n})$. If $u_{2}=a_{2}$, then $\left\{f_{1}, f_{2}, x_{4}^{u_{4}}-x_{1}^{u_{1}+a_{1}}\right\}$ is a standard basis for $I(\mathbf{n})$ with respect to the negative degree reverse lexicographical order with $x_{3}>x_{4}>x_{2}>x_{1}$. By [8, Lemma 5.5.11] $I(\mathbf{n})_{*}$ is a complete intersection. Assume that $u_{2}<a_{2}$. We will show that $u_{4} \leq u_{1}+u_{2}$. Suppose that $u_{4}>u_{1}+u_{2}$, then $x_{2}^{u_{2}} \in I(\mathbf{n})_{*}:\left\langle x_{1}\right\rangle$ and therefore $x_{2}^{u_{2}} \in I(\mathbf{n})_{*}$. Thus $x_{2}^{u_{2}}$ is divided by $x_{2}^{a_{2}}$, a contradiction. Then $\left\{f_{1}, f_{2}, f_{3}\right\}$ is a standard basis for $I(\mathbf{n})$ with respect to the negative degree reverse lexicographical order with $x_{3}>x_{4}>x_{2}>x_{1}$. Note that $\operatorname{LM}\left(f_{1}\right)=x_{2}^{a_{2}}, \operatorname{LM}\left(f_{2}\right)=$ $x_{3}^{a_{3}}$ and $\operatorname{LM}\left(f_{3}\right)=x_{4}^{u_{4}}$. By [8, Lemma 5.5.11] $I(\mathbf{n})_{*}$ is a complete intersection. Suppose now that $u_{4}=0$, so necessarily $u_{3}=a_{3}$. Then $\left\{f_{1}, f_{2}, f_{4}=x_{4}^{a_{4}}-x_{1}^{u_{1}} x_{2}^{u_{2}}\right\}$ is a minimal generating set of $I(\mathbf{n})$. If $u_{2}=a_{2}$, then $\left\{f_{1}, f_{2}, x_{4}^{a_{4}}-x_{1}^{a_{1}+u_{1}}\right\}$ is a standard basis for $I(\mathbf{n})$ with respect to the negative degree reverse lexicographical order with $x_{3}>x_{4}>x_{2}>x_{1}$. Thus, from [8, Lemma 5.5.11], $I(\mathbf{n})_{*}$ is a complete intersection. Assume that $u_{2}<a_{2}$, then $a_{4} \leq u_{1}+u_{2}$ and also $\left\{f_{1}, f_{2}, f_{4}\right\}$ is a standard basis for $I(\mathbf{n})$ with respect to the negative degree reverse lexicographical order with $x_{3}>x_{4}>x_{2}>x_{1}$. From [8, Lemma 5.5.11] we deduce that $I(\mathbf{n})_{*}$ is a complete intersection.

Remark 2.4. In case (B) the minimal number of generators of $I(\mathbf{n})_{*}$ can be arbitrarily large even if the tangent cone of $C(\mathbf{n})$ is Cohen-Macaulay, see [14, Proposition 3.14].

Given a complete intersection monomial curve $C(\mathbf{n})$, we next study the complete intersection property of $C(\mathbf{n}+w \mathbf{v})$. Let $M$ be a non-zero $r \times s$ integer matrix, then there exist an $r \times r$ invertible integer matrix $U$ and an $s \times s$ invertible integer matrix $V$ such that $U M V=\operatorname{diag}\left(\delta_{1}, \ldots, \delta_{m}, 0, \ldots, 0\right)$ is the diagonal matrix, where $\delta_{j}$ for all $j=1,2, \ldots, m$ are positive integers such that $\delta_{i} \mid \delta_{i+1}, 1 \leq i \leq m-1$, and $m$ is the rank of $M$. The elements $\delta_{1}, \ldots, \delta_{m}$ are the invariant factors of $M$. By [9, Theorem 3.9] the product $\delta_{1} \delta_{2} \cdots \delta_{m}$ equals the greatest common divisor of all non-zero $m \times m$ minors of $M$.

The following proposition will be useful in the proof of Theorem 2.6.

Proposition 2.5. Let $B=\left\{f_{1}=x_{1}^{b_{1}}-x_{2}^{b_{2}}, f_{2}=x_{3}^{b_{3}}-x_{4}^{b_{4}}, f_{3}=x_{1}^{v_{1}} x_{2}^{v_{2}}-x_{3}^{v_{3}} x_{4}^{v_{4}}\right\}$ be a set of binomials in $K\left[x_{1}, \ldots, x_{4}\right]$, where $b_{i} \geq 1$ for all $1 \leq i \leq 4$, at least one of $v_{1}$, 
$v_{2}$ is non-zero and at least one of $v_{3}, v_{4}$ is non-zero. Let $n_{1}=b_{2}\left(b_{3} v_{4}+v_{3} b_{4}\right), n_{2}=$ $b_{1}\left(b_{3} v_{4}+v_{3} b_{4}\right), n_{3}=b_{4}\left(b_{1} v_{2}+v_{1} b_{2}\right), n_{4}=b_{3}\left(b_{1} v_{2}+v_{1} b_{2}\right)$. If $\operatorname{gcd}\left(n_{1}, \ldots, n_{4}\right)=1$, then $I(\mathbf{n})$ is a complete intersection ideal generated by the binomials $f_{1}, f_{2}$ and $f_{3}$.

Proof. Consider the vectors $\mathbf{d}_{1}=\left(b_{1},-b_{2}, 0,0\right), \mathbf{d}_{2}=\left(0,0, b_{3},-b_{4}\right)$ and $\mathbf{d}_{3}=$ $\left(v_{1}, v_{2},-v_{3},-v_{4}\right)$. Clearly $\mathbf{d}_{i} \in \operatorname{ker}_{\mathbb{Z}}\left(n_{1}, \ldots, n_{4}\right)$ for $1 \leq i \leq 3$, so the lattice $L=\sum_{i=1}^{3} \mathbb{Z} \mathbf{d}_{i}$ is a subset of $\operatorname{ker}_{\mathbb{Z}}\left(n_{1}, \ldots, n_{4}\right)$. Consider the matrix

$$
M=\left(\begin{array}{ccc}
b_{1} & 0 & v_{1} \\
-b_{2} & 0 & v_{2} \\
0 & b_{3} & -v_{3} \\
0 & -b_{4} & -v_{4}
\end{array}\right)
$$

It is not hard to show that the rank of $M$ equals 3 . We will prove that $L$ is saturated, namely the invariant factors $\delta_{1}, \delta_{2}$ and $\delta_{3}$ of $M$ are all equal to 1 . The greatest common divisor of all non-zero $3 \times 3$ minors of $M$ equals the greatest common divisor of the integers $n_{1}, n_{2}, n_{3}$ and $n_{4}$. $\operatorname{But} \operatorname{gcd}\left(n_{1}, \ldots, n_{4}\right)=1$, so $\delta_{1} \delta_{2} \delta_{3}=1$ and therefore $\delta_{1}=\delta_{2}=\delta_{3}=1$. Note that the rank of the lattice $\operatorname{ker}_{\mathbb{Z}}\left(n_{1}, \ldots, n_{4}\right)$ is 3 and also equals the rank of $L$. By [17, Lemma 8.2.5] we have that $L=\operatorname{ker}_{\mathbb{Z}}\left(n_{1}, \ldots, n_{4}\right)$. Now the transpose $M^{t}$ of $M$ is mixed dominating. Recall that a matrix $P$ is mixed dominating if every row of $P$ has a positive and negative entry and $P$ contains no square submatrix with this property. By [5, Theorem 2.9] $I(\mathbf{n})$ is a complete intersection on the binomials $f_{1}, f_{2}$ and $f_{3}$.

Theorem 2.6. Let $I(\mathbf{n})$ be a complete intersection ideal generated by the binomials $f_{1}=x_{1}^{a_{1}}-x_{2}^{a_{2}}, f_{2}=x_{3}^{a_{3}}-x_{4}^{a_{4}}$ and $f_{3}=x_{1}^{u_{1}} x_{2}^{u_{2}}-x_{3}^{u_{3}} x_{4}^{u_{4}}$. Then there exist vectors $\mathbf{v}_{i}, 1 \leq i \leq 22$, in $\mathbb{N}^{4}$ such that for all $w>0$, the toric ideal $I\left(\mathbf{n}+w \mathbf{v}_{i}\right)$ is a complete intersection whenever the entries of $\mathbf{n}+w \mathbf{v}_{i}$ are relatively prime.

Proof. By [11, Theorem 6] $n_{1}=a_{2}\left(a_{3} u_{4}+u_{3} a_{4}\right), n_{2}=a_{1}\left(a_{3} u_{4}+u_{3} a_{4}\right), n_{3}=$ $a_{4}\left(a_{1} u_{2}+u_{1} a_{2}\right), n_{4}=a_{3}\left(a_{1} u_{2}+u_{1} a_{2}\right)$. Let $\mathbf{v}_{1}=\left(a_{2} a_{3}, a_{1} a_{3}, a_{2} a_{4}, a_{2} a_{3}\right)$ and $B=$ $\left\{f_{1}, f_{2}, f_{4}=x_{1}^{u_{1}+w} x_{2}^{u_{2}}-x_{3}^{u_{3}} x_{4}^{u_{4}+w}\right\}$. Then $n_{1}+w a_{2} a_{3}=a_{2}\left(a_{3}\left(u_{4}+w\right)+u_{3} a_{4}\right), n_{2}+$ $w a_{1} a_{3}=a_{1}\left(a_{3}\left(u_{4}+w\right)+u_{3} a_{4}\right), n_{3}+w a_{2} a_{4}=a_{4}\left(a_{1} u_{2}+\left(u_{1}+w\right) a_{2}\right)$ and $n_{4}+w a_{2} a_{3}=$ $a_{3}\left(a_{1} u_{2}+\left(u_{1}+w\right) a_{2}\right)$. By Proposition 2.5 for every $w>0$, the ideal $I\left(\mathbf{n}+w \mathbf{v}_{1}\right)$ is a complete intersection on $f_{1}, f_{2}$ and $f_{4}$ whenever $\operatorname{gcd}\left(n_{1}+w a_{2} a_{3}, n_{2}+w a_{1} a_{3}, n_{3}+\right.$ $\left.w a_{2} a_{4}, n_{4}+w a_{2} a_{3}\right)=1$. Consider the vectors $\mathbf{v}_{2}=\left(a_{2} a_{3}, a_{1} a_{3}, a_{1} a_{4}, a_{1} a_{3}\right), \mathbf{v}_{3}=$ $\left(a_{2} a_{4}, a_{1} a_{4}, a_{2} a_{4}, a_{2} a_{3}\right), \mathbf{v}_{4}=\left(a_{2} a_{4}, a_{1} a_{4}, a_{1} a_{4}, a_{1} a_{3}\right), \mathbf{v}_{5}=\left(a_{2}\left(a_{3}+a_{4}\right), a_{1}\left(a_{3}+\right.\right.$ $\left.\left.a_{4}\right), 0,0\right)$ and $\mathbf{v}_{6}=\left(0,0, a_{4}\left(a_{1}+a_{2}\right), a_{3}\left(a_{1}+a_{2}\right)\right)$. By Proposition 2.5 for every $w>0$, $I\left(\mathbf{n}+w \mathbf{v}_{2}\right)$ is a complete intersection on $f_{1}, f_{2}$ and $x_{1}^{u_{1}} x_{2}^{u_{2}+w}-x_{3}^{u_{3}} x_{4}^{u_{4}+w}$ whenever the entries of $\mathbf{n}+w \mathbf{v}_{2}$ are relatively prime, $I\left(\mathbf{n}+w \mathbf{v}_{3}\right)$ is a complete intersection on $f_{1}, f_{2}$ and $x_{1}^{u_{1}+w} x_{2}^{u_{2}}-x_{3}^{u_{3}+w} x_{4}^{u_{4}}$ whenever the entries of $\mathbf{n}+w \mathbf{v}_{3}$ are relatively prime, and $I\left(\mathbf{n}+w \mathbf{v}_{4}\right)$ is a complete intersection on $f_{1}, f_{2}$ and $x_{1}^{u_{1}} x_{2}^{u_{2}+w}-x_{3}^{u_{3}+w} x_{4}^{u_{4}}$ whenever the entries of $\mathbf{n}+w \mathbf{v}_{4}$ are relatively prime. Furthermore for all $w>0$, $I\left(\mathbf{n}+w \mathbf{v}_{5}\right)$ is a complete intersection on $f_{1}, f_{2}$ and $x_{1}^{u_{1}} x_{2}^{u_{2}}-x_{3}^{u_{3}+w} x_{4}^{u_{4}+w}$ whenever the entries of $\mathbf{n}+w \mathbf{v}_{5}$ are relatively prime, and $I\left(\mathbf{n}+w \mathbf{v}_{6}\right)$ is a complete intersection on $f_{1}, f_{2}$ and $x_{1}^{u_{1}+w} x_{2}^{u_{2}+w}-x_{3}^{u_{3}} x_{4}^{u_{4}}$ whenever the entries of $\mathbf{n}+w \mathbf{v}_{6}$ are relatively prime. Consider the vectors $\mathbf{v}_{7}=\left(a_{2}\left(a_{3}+a_{4}\right), a_{1}\left(a_{3}+a_{4}\right), a_{2} a_{4}, a_{2} a_{3}\right)$, $\mathbf{v}_{8}=\left(a_{2}\left(a_{3}+a_{4}\right), a_{1}\left(a_{3}+a_{4}\right), a_{4}\left(a_{1}+a_{2}\right), a_{3}\left(a_{1}+a_{2}\right)\right), \mathbf{v}_{9}=\left(0,0, a_{2} a_{4}, a_{2} a_{3}\right)$, $\mathbf{v}_{10}=\left(a_{2} a_{4}, a_{1} a_{4}, a_{4}\left(a_{1}+a_{2}\right), a_{3}\left(a_{1}+a_{2}\right)\right), \mathbf{v}_{11}=\left(a_{2} a_{3}, a_{1} a_{3}, a_{4}\left(a_{1}+a_{2}\right), a_{3}\left(a_{1}+\right.\right.$ $\left.\left.a_{2}\right)\right), \mathbf{v}_{12}=\left(a_{2}\left(a_{3}+a_{4}\right), a_{1}\left(a_{3}+a_{4}\right), a_{1} a_{4}, a_{1} a_{3}\right), \mathbf{v}_{13}=\left(0,0, a_{1} a_{4}, a_{1} a_{3}\right), \mathbf{v}_{14}=$ $\left(a_{2} a_{4}, a_{1} a_{4}, 0,0\right)$ and $\mathbf{v}_{15}=\left(a_{2} a_{3}, a_{1} a_{3}, 0,0\right)$. Using Proposition 2.5 we have that for all $w>0, I\left(\mathbf{n}+w \mathbf{v}_{i}\right), 7 \leq i \leq 15$, is a complete intersection whenever the entries of $\mathbf{n}+w \mathbf{v}_{i}$ are relatively prime. For instance $I\left(\mathbf{n}+w \mathbf{v}_{9}\right)$ is a complete intersection on the binomials $f_{1}, f_{2}$ and $x_{1}^{u_{1}+w} x_{2}^{u_{2}}-x_{3}^{u_{3}} x_{4}^{u_{4}}$. Consider the vectors $\mathbf{v}_{16}=\left(a_{3} u_{4}+u_{3} a_{4}, a_{3} u_{4}+u_{3} a_{4}, a_{4}\left(u_{1}+u_{2}\right), a_{3}\left(u_{1}+u_{2}\right)\right), \mathbf{v}_{17}=\left(0, a_{3} u_{4}+\right.$ 
$\left.u_{3} a_{4}, u_{2} a_{4}, u_{2} a_{3}\right), \mathbf{v}_{18}=\left(a_{3} u_{4}+u_{3} a_{4}, 0, u_{1} a_{4}, u_{1} a_{3}\right), \mathbf{v}_{19}=\left(a_{2} u_{4}, a_{1} u_{4}, 0, a_{1} u_{2}+\right.$ $\left.u_{1} a_{2}\right), \mathbf{v}_{20}=\left(a_{2} u_{3}, a_{1} u_{3}, a_{1} u_{2}+u_{1} a_{2}, 0\right), \mathbf{v}_{21}=\left(a_{2}\left(a_{4}+u_{4}\right), a_{1}\left(a_{4}+u_{4}\right), 0, a_{1} u_{2}+\right.$ $\left.u_{1} a_{2}\right)$ and $\mathbf{v}_{22}=\left(a_{2}\left(u_{3}+u_{4}\right), a_{1}\left(u_{3}+u_{4}\right), a_{1} u_{2}+u_{1} a_{2}, a_{1} u_{2}+u_{1} a_{2}\right)$. It is easy to see that for all $w>0$, the ideal $I\left(\mathbf{n}+w \mathbf{v}_{i}\right), 16 \leq i \leq 22$, is a complete intersection whenever the entries of $\mathbf{n}+w \mathbf{v}_{i}$ are relatively prime. For instance $I\left(\mathbf{n}+w \mathbf{v}_{16}\right)$ is a complete intersection on the binomials $f_{2}, f_{3}$ and $x_{1}^{a_{1}+w}-x_{2}^{a_{2}+w}$.

Example 2.7. Let $\mathbf{n}=(93,124,195,117)$, then $I(\mathbf{n})$ is a complete intersection on the binomials $x_{1}^{4}-x_{2}^{3}, x_{3}^{3}-x_{4}^{5}$ and $x_{1}^{9} x_{2}^{3}-x_{3}^{2} x_{4}^{7}$. Here $a_{1}=4, a_{2}=3, a_{3}=3$, $a_{4}=5, u_{1}=9, u_{2}=3, u_{3}=2$ and $u_{4}=7$. Consider the vector $\mathbf{v}_{1}=(9,12,15,9)$. For all $w \geq 0$ the ideal $I\left(\mathbf{n}+w \mathbf{v}_{1}\right)$ is a complete intersection on $x_{1}^{4}-x_{2}^{3}, x_{3}^{3}-x_{4}^{5}$ and $x_{1}^{9+w} x_{2}^{3}-x_{3}^{2} x_{4}^{w+7}$ whenever $\operatorname{gcd}(93+9 w, 124+12 w, 195+15 w, 117+9 w)=1$. Ву Corollary 2.2 the monomial curve $C\left(\mathbf{n}+w \mathbf{v}_{1}\right)$ has Cohen-Macaulay tangent cone at the origin. Consider the vector $\mathbf{v}_{4}=(15,20,20,12)$ and the sequence $\mathbf{n}+9 \mathbf{v}_{4}=$ $(228,304,375,225)$. The toric ideal $I\left(\mathbf{n}+9 \mathbf{v}_{4}\right)$ is a complete intersection on the binomials $x_{1}^{4}-x_{2}^{3}, x_{3}^{3}-x_{4}^{5}$ and $x_{1}^{21} x_{2}^{3}-x_{3}^{2} x_{4}^{22}$. Note that $x_{1}^{25}-x_{3}^{2} x_{4}^{22} \in I\left(\mathbf{n}+9 \mathbf{v}_{4}\right)$, so $x_{3}^{2} x_{4}^{22} \in I\left(\mathbf{n}+9 \mathbf{v}_{4}\right)_{*}$ and also $x_{3}^{2} \in I\left(\mathbf{n}+9 \mathbf{v}_{4}\right)_{*}:\left\langle x_{4}\right\rangle$. If $C\left(\mathbf{n}+9 \mathbf{v}_{4}\right)$ has CohenMacaulay tangent cone at the origin, then $x_{3}^{2} \in I\left(\mathbf{n}+9 \mathbf{v}_{4}\right)_{*}$ a contradiction. Thus $C\left(\mathbf{n}+9 \mathbf{v}_{4}\right)$ does not have a Cohen-Macaulay tangent cone at the origin.

Theorem 2.8. Let $I(\mathbf{n})$ be a complete intersection ideal generated by the binomials $f_{1}=x_{1}^{a_{1}}-x_{2}^{a_{2}}, f_{2}=x_{3}^{a_{3}}-x_{4}^{a_{4}}$ and $f_{3}=x_{1}^{u_{1}} x_{2}^{u_{2}}-x_{3}^{u_{3}} x_{4}^{u_{4}}$. Consider the vector $\mathbf{d}=\left(a_{2} a_{3}, a_{1} a_{3}, a_{2} a_{4}, a_{2} a_{3}\right)$. If $C(\mathbf{n})$ has Cohen-Macaulay tangent cone at the origin, then for every $w>0$ the monomial curve $C(\mathbf{n}+w \mathbf{d})$ has Cohen-Macaulay tangent cone at the origin whenever the entries of $\mathbf{n}+w \mathbf{d}$ are relatively prime.

Proof. Let $n_{1}=\min \left\{n_{1}, \ldots, n_{4}\right\}$ and also $a_{3}<a_{4}$. Without loss of generality we can assume that $u_{2} \leq a_{2}$ and $u_{3} \leq a_{3}$. By Theorem 2.6 for every $w>0$, the ideal $I(\mathbf{n}+w \mathbf{d})$ is a complete intersection on $f_{1}, f_{2}$ and $f_{4}=x_{1}^{u_{1}+w} x_{2}^{u_{2}}-x_{3}^{u_{3}} x_{4}^{u_{4}+w}$ whenever the entries of $\mathbf{n}+w \mathbf{d}$ are relatively prime. Note that $n_{1}+w a_{2} a_{3}=$ $\min \left\{n_{1}+w a_{2} a_{3}, n_{2}+w a_{1} a_{3}, n_{3}+w a_{2} a_{4}, n_{4}+w a_{2} a_{3}\right\}$. Suppose that $u_{3}>0$ and $u_{4}>$ 0 . Assume that $u_{2}<a_{2}$. By Corollary 2.2 it holds that $a_{4}+u_{4} \leq u_{1}+u_{2}+a_{3}-u_{3}$ and therefore

$$
a_{4}+\left(u_{4}+w\right) \leq\left(u_{1}+w\right)+u_{2}+a_{3}-u_{3} .
$$

Thus, from Corollary 2.2 again $C(\mathbf{n}+w \mathbf{d})$ has Cohen-Macaulay tangent cone at the origin. Assume that $u_{2}=a_{2}$. Then, from Corollary 2.2 we have that $a_{4}+u_{4} \leq$ $u_{1}+a_{1}+a_{3}-u_{3}$ and therefore $a_{4}+\left(u_{4}+w\right) \leq\left(u_{1}+w\right)+a_{1}+a_{3}-u_{3}$. By Corollary $2.2 C(\mathbf{n}+w \mathbf{d})$ has Cohen-Macaulay tangent cone at the origin.

Suppose now that $u_{3}=0$. Then $\left\{f_{1}, f_{2}, f_{5}=x_{4}^{u_{4}+w}-x_{1}^{u_{1}+w} x_{2}^{u_{2}}\right\}$ is a minimal generating set of $I(\mathbf{n}+w \mathbf{d})$. If $u_{2}=a_{2}$, then $\left\{f_{1}, f_{2}, x_{4}^{u_{4}+w}-x_{1}^{u_{1}+a_{1}+w}\right\}$ is a standard basis for $I(\mathbf{n}+w \mathbf{d})$ with respect to the negative degree reverse lexicographical order with $x_{3}>x_{4}>x_{2}>x_{1}$. Thus $I(\mathbf{n}+w \mathbf{d})_{*}$ is a complete intersection and therefore $C(\mathbf{n}+w \mathbf{d})$ has Cohen-Macaulay tangent cone at the origin. Assume that $u_{2}<a_{2}$, then $u_{4} \leq u_{1}+u_{2}$ and therefore $u_{4}+w \leq\left(u_{1}+w\right)+u_{2}$. The set $\left\{f_{1}, f_{2}, f_{5}\right\}$ is a standard basis for $I(\mathbf{n}+w \mathbf{d})$ with respect to the negative degree reverse lexicographical order with $x_{3}>x_{4}>x_{2}>x_{1}$. Thus $I(\mathbf{n}+w \mathbf{d})_{*}$ is a complete intersection and therefore $C(\mathbf{n}+w \mathbf{d})$ has Cohen-Macaulay tangent cone at the origin.

Suppose that $u_{4}=0$, so necessarily $u_{3}=a_{3}$. Then $\left\{f_{1}, f_{2}, x_{4}^{a_{4}+w}-x_{1}^{u_{1}+w} x_{2}^{u_{2}}\right\}$ is a minimal generating set of $I(\mathbf{n}+w \mathbf{d})$. If $u_{2}=a_{2}$, then $\left\{f_{1}, f_{2}, x_{4}^{a_{4}+w}-x_{1}^{u_{1}+a_{1}+w}\right\}$ is a standard basis for $I(\mathbf{n}+w \mathbf{d})$ with respect to the negative degree reverse lexicographical order with $x_{3}>x_{4}>x_{2}>x_{1}$. Thus $I(\mathbf{n}+w \mathbf{d})_{*}$ is a complete intersection and therefore $C(\mathbf{n}+w \mathbf{d})$ has Cohen-Macaulay tangent cone at the origin. Assume that $u_{2}<a_{2}$, then $a_{4} \leq u_{1}+u_{2}$ and therefore $a_{4}+w \leq\left(u_{1}+w\right)+u_{2}$. The set $\left\{f_{1}, f_{2}, x_{4}^{a_{4}+w}-x_{1}^{u_{1}+w} x_{2}^{u_{2}}\right\}$ is a standard basis for $I(\mathbf{n}+w \mathbf{d})$ with respect to 
the negative degree reverse lexicographical order with $x_{3}>x_{4}>x_{2}>x_{1}$. Thus $I(\mathbf{n}+w \mathbf{d})_{*}$ is a complete intersection and therefore $C(\mathbf{n}+w \mathbf{d})$ has Cohen-Macaulay tangent cone at the origin.

Theorem 2.9. Let $I(\mathbf{n})$ be a complete intersection ideal generated by the binomials $f_{1}=x_{1}^{a_{1}}-x_{2}^{a_{2}}, f_{2}=x_{3}^{a_{3}}-x_{4}^{a_{4}}$ and $f_{3}=x_{1}^{u_{1}} x_{2}^{u_{2}}-x_{3}^{u_{3}} x_{4}^{u_{4}}$. Consider the vectors $\mathbf{d}_{1}=\left(0,0, a_{2} a_{4}, a_{2} a_{3}\right)$ and $\mathbf{d}_{2}=\left(0,0, a_{1} a_{4}, a_{1} a_{3}\right)$. Then there exists a non-negative integer $w_{0}$ such that for all $w \geq w_{0}$, the monomial curves $C\left(\mathbf{n}+w \mathbf{d}_{1}\right)$ and $C(\mathbf{n}+$ $\left.w \mathbf{d}_{2}\right)$ have Cohen-Macaulay tangent cones at the origin whenever the entries of the corresponding sequence $\left(\mathbf{n}+w \mathbf{d}_{1}\right.$ for the first family and $\mathbf{n}+w \mathbf{d}_{2}$ for the second) are relatively prime.

Proof. Let $n_{1}=\min \left\{n_{1}, \ldots, n_{4}\right\}$ and $a_{3}<a_{4}$. Suppose that $u_{2} \leq a_{2}$ and $u_{3} \leq a_{3}$. By Theorem 2.6 for all $w \geq 0, I\left(\mathbf{n}+w \mathbf{d}_{1}\right)$ is a complete intersection on $f_{1}, f_{2}$ and $f_{4}=x_{1}^{u_{1}+w} x_{2}^{u_{2}}-x_{3}^{u_{3}} x_{4}^{u_{4}}$ whenever the entries of $\mathbf{n}+w \mathbf{d}_{\mathbf{1}}$ are relatively prime. Remark that $n_{1}=\min \left\{n_{1}, n_{2}, n_{3}+w a_{2} a_{4}, n_{4}+w a_{2} a_{3}\right\}$. Let $w_{0}$ be the smallest non-negative integer greater than or equal to $u_{3}+u_{4}-$ $u_{1}-u_{2}+a_{4}-a_{3}$. Then for every $w \geq w_{0}$ we have that $a_{4}+u_{4} \leq u_{1}+w+$ $u_{2}+a_{3}-u_{3}$, so $u_{3}+u_{4}<u_{1}+w+u_{2}$. Let $G=\left\{f_{1}, f_{2}, f_{4}, f_{5}=x_{4}^{a_{4}+u_{4}}-\right.$ $\left.x_{1}^{u_{1}+w} x_{2}^{u_{2}} x_{3}^{a_{3}-u_{3}}\right\}$. We will prove that for every $w \geq w_{0}, G$ is a standard basis for $I\left(\mathbf{n}+w \mathbf{d}_{1}\right)$ with respect to the negative degree reverse lexicographical order with $x_{3}>x_{4}>x_{2}>x_{1}$. Note that $\operatorname{LM}\left(f_{1}\right)=x_{2}^{a_{2}}, \operatorname{LM}\left(f_{2}\right)=x_{3}^{a_{3}}, \operatorname{LM}\left(f_{4}\right)=$ $x_{3}^{u_{3}} x_{4}^{u_{4}}$ and $\operatorname{LM}\left(f_{5}\right)=x_{4}^{a_{4}+u_{4}}$. Therefore $\operatorname{NF}\left(\operatorname{spoly}\left(f_{i}, f_{j}\right) \mid G\right)=0$ as $\operatorname{LM}\left(f_{i}\right)$ and $\operatorname{LM}\left(f_{j}\right)$ are relatively prime, for $(i, j) \in\{(1,2),(1,4),(1,5),(2,5)\}$. We compute $\operatorname{spoly}\left(f_{2}, f_{4}\right)=-f_{5}, \operatorname{so} \operatorname{NF}\left(\operatorname{spoly}\left(f_{2}, f_{4}\right) \mid G\right)=0$. Next we compute $\operatorname{spoly}\left(f_{4}, f_{5}\right)=$ $x_{1}^{u_{1}+w} x_{2}^{u_{2}} x_{3}^{a_{3}}-x_{1}^{u_{1}+w} x_{2}^{u_{2}} x_{4}^{a_{4}}$. Then $\operatorname{LM}\left(\operatorname{spoly}\left(f_{4}, f_{5}\right)\right)=x_{1}^{u_{1}+w} x_{2}^{u_{2}} x_{3}^{a_{3}}$ and only $\operatorname{LM}\left(f_{2}\right)$ divides $\operatorname{LM}\left(\operatorname{spoly}\left(f_{4}, f_{5}\right)\right)$. Also ecart $\left(\operatorname{spoly}\left(f_{4}, f_{5}\right)\right)=a_{4}-a_{3}=\operatorname{ecart}\left(f_{2}\right)$. Then $\operatorname{spoly}\left(f_{2}, \operatorname{spoly}\left(f_{4}, f_{5}\right)\right)=0$ and $\operatorname{NF}\left(\operatorname{spoly}\left(f_{4}, f_{5}\right) \mid G\right)=0$. Thus the minimal number of generators for $I\left(\mathbf{n}+w \mathbf{d}_{1}\right)_{*}$ is either three or four, so from [14, Theorem 3.1] for every $w \geq w_{0}, C\left(\mathbf{n}+w \mathbf{d}_{1}\right)$ has Cohen-Macaulay tangent cone at the origin whenever the entries of $\mathbf{n}+w \mathbf{d}_{1}$ are relatively prime.

By Theorem 2.6 for all $w \geq 0, I\left(\mathbf{n}+w \mathbf{d}_{2}\right)$ is a complete intersection on $f_{1}, f_{2}$ and $f_{6}=x_{1}^{u_{1}} x_{2}^{u_{2}+w}-x_{3}^{u_{3}} x_{4}^{u_{4}}$ whenever the entries of $\mathbf{n}+w \mathbf{d}_{2}$ are relatively prime. Remark that $n_{1}=\min \left\{n_{1}, n_{2}, n_{3}+w a_{1} a_{4}, n_{4}+w a_{1} a_{3}\right\}$. For every $w \geq w_{0}$ the set $H=\left\{f_{1}, f_{2}, f_{6}, x_{4}^{a_{4}+u_{4}}-x_{1}^{u_{1}} x_{2}^{u_{2}+w} x_{3}^{a_{3}-u_{3}}\right\}$ is a standard basis for $I\left(\mathbf{n}+w \mathbf{d}_{2}\right)$ with respect to the negative degree reverse lexicographical order with $x_{3}>x_{4}>x_{2}>x_{1}$. Thus the minimal number of generators for $I\left(\mathbf{n}+w \mathbf{d}_{2}\right)_{*}$ is either three or four, so from [14, Theorem 3.1] for every $w \geq w_{0}, C\left(\mathbf{n}+w \mathbf{d}_{2}\right)$ has Cohen-Macaulay tangent cone at the origin whenever the entries of $\mathbf{n}+w \mathbf{d}_{2}$ are relatively prime.

Example 2.10. Let $\mathbf{n}=(15,25,24,16)$, then $I(\mathbf{n})$ is a complete intersection on the binomials $x_{1}^{5}-x_{2}^{3}, x_{3}^{2}-x_{4}^{3}$ and $x_{1} x_{2}-x_{3} x_{4}$. Here $a_{1}=5, a_{2}=3, a_{3}=2, a_{4}=3$, $u_{i}=1,1 \leq i \leq 4$. Note that $x_{4}^{4}-x_{1} x_{2} x_{3} \in I(\mathbf{n})$, so, from Corollary 2.2 $C(\mathbf{n})$ does not have a Cohen-Macaulay tangent cone at the origin. Consider the vector $\mathbf{d}_{1}=$ $(0,0,9,6)$. For every $w>0$ the ideal $I\left(\mathbf{n}+w \mathbf{d}_{1}\right)$ is a complete intersection on the binomials $x_{1}^{5}-x_{2}^{3}, x_{3}^{2}-x_{4}^{3}$ and $x_{1}^{w+1} x_{2}-x_{3} x_{4}$ whenever $\operatorname{gcd}(15,25,24+9 w, 16+6 w)=$ 1. By Theorem 2.9 for every $w \geq 1$, the monomial curve $C\left(\mathbf{n}+w \mathbf{d}_{1}\right)$ has CohenMacaulay tangent cone at the origin whenever $\operatorname{gcd}(15,25,24+9 w, 16+6 w)=1$.

The next example gives a family of complete intersection monomial curves supporting M. Rossi's problem, although their tangent cones are not Cohen-Macaulay. To prove it we will use the following proposition.

Proposition 2.11. 22, Proposition 2.2] Let $I \subset K\left[x_{1}, x_{2}, \ldots, x_{d}\right]$ be a monomial ideal and $I=\left\langle J, \mathbf{x}^{\mathbf{u}}\right\rangle$ for a monomial ideal $J$ and a monomial $\mathbf{x}^{\mathbf{u}}$. Let $p(I)$ denote 
the numerator $g(t)$ of the Hilbert Series for $K\left[x_{1}, x_{2}, \ldots, x_{d}\right] / I$. Then $p(I)=p(J)-$ $t^{\operatorname{deg}\left(\mathbf{x}^{\mathbf{u}}\right)} p\left(J:\left\langle\mathbf{x}^{\mathbf{u}}\right\rangle\right)$.

Example 2.12. Consider the family $n_{1}=8 m^{2}+6, n_{2}=20 m^{2}+15, n_{3}=12 m^{2}+15$ and $n_{4}=8 m^{2}+10$, where $m \geq 1$ is an integer. The toric ideal $I(\mathbf{n})$ is minimally generated by the binomials

$$
x_{1}^{5}-x_{2}^{2}, x_{3}^{2}-x_{4}^{3}, x_{1}^{2 m^{2}} x_{2}-x_{3} x_{4}^{2 m^{2}} .
$$

Consider the vector $\mathbf{v}_{1}=(4,10,6,4)$ and the family $n_{1}^{\prime}=n_{1}+4 w, n_{2}^{\prime}=n_{2}+10 w$, $n_{3}^{\prime}=n_{3}+6 w, n_{4}^{\prime}=n_{4}+4 w$ where $w \geq 0$ is an integer. Let $\mathbf{n}^{\prime}=\left(n_{1}^{\prime}, n_{2}^{\prime}, n_{3}^{\prime}, n_{4}^{\prime}\right)$, then for all $w \geq 0$ the toric ideal $I\left(\mathbf{n}^{\prime}\right)$ is minimally generated by the binomials

$$
x_{1}^{5}-x_{2}^{2}, x_{3}^{2}-x_{4}^{3}, x_{1}^{2 m^{2}+w} x_{2}-x_{3} x_{4}^{2 m^{2}+w}
$$

whenever $\operatorname{gcd}\left(n_{1}^{\prime}, n_{2}^{\prime}, n_{3}^{\prime}, n_{4}^{\prime}\right)=1$. Let $C_{m}\left(\mathbf{n}^{\prime}\right)$ be the corresponding monomial curve. By Corollary 2.2 for all $w \geq 0$, the monomial curve $C_{m}\left(\mathbf{n}^{\prime}\right)$ does not have CohenMacaulay tangent cone at the origin whenever $\operatorname{gcd}\left(n_{1}^{\prime}, n_{2}^{\prime}, n_{3}^{\prime}, n_{4}^{\prime}\right)=1$. We will show that for every $w \geq 0$, the Hilbert function of the $\operatorname{ring} K\left[\left[t^{n_{1}^{\prime}}, \ldots, t^{n_{4}^{\prime}}\right]\right]$ is non-decreasing whenever $\operatorname{gcd}\left(n_{1}^{\prime}, n_{2}^{\prime}, n_{3}^{\prime}, n_{4}^{\prime}\right)=1$. It suffices to prove that for every $w \geq 0$, the Hilbert function of $K\left[x_{1}, x_{2}, x_{3}, x_{4}\right] / I\left(\mathbf{n}^{\prime}\right)_{*}$ is non-decreasing whenever $\operatorname{gcd}\left(n_{1}^{\prime}, n_{2}^{\prime}, n_{3}^{\prime}, n_{4}^{\prime}\right)=1$. The set

$$
\begin{gathered}
G=\left\{x_{1}^{5}-x_{2}^{2}, x_{3}^{2}-x_{4}^{3}, x_{1}^{2 m^{2}+w} x_{2}-x_{3} x_{4}^{2 m^{2}+w}, x_{4}^{2 m^{2}+w+3}-x_{1}^{2 m^{2}+w} x_{2} x_{3},\right. \\
\left.x_{1}^{2 m^{2}+w+5} x_{3}-x_{2} x_{4}^{2 m^{2}+w+3}, x_{1}^{4 m^{2}+2 w+5}-x_{4}^{4 m^{2}+2 w+3}\right\}
\end{gathered}
$$

is a standard basis for $I\left(\mathbf{n}^{\prime}\right)$ with respect to the negative degree reverse lexicographical order with $x_{4}>x_{3}>x_{2}>x_{1}$. Thus $I\left(\mathbf{n}^{\prime}\right)_{*}$ is generated by the set

$$
\left\{x_{2}^{2}, x_{3}^{2}, x_{4}^{4 m^{2}+2 w+3}, x_{1}^{2 m^{2}+w} x_{2} x_{3}, x_{1}^{2 m^{2}+w} x_{2}-x_{3} x_{4}^{2 m^{2}+w}, x_{2} x_{4}^{2 m^{2}+w+3}\right\} .
$$

Also $\left\langle\operatorname{LT}\left(I\left(\mathbf{n}^{\prime}\right)_{*}\right)\right\rangle$ with respect to the aforementioned order can be written as,

$$
\left\langle\operatorname{LT}\left(I\left(\mathbf{n}^{\prime}\right)_{*}\right)\right\rangle=\left\langle x_{2}^{2}, x_{3}^{2}, x_{4}^{4 m^{2}+2 w+3}, x_{2} x_{4}^{2 m^{2}+w+3}, x_{3} x_{4}^{2 m^{2}+w}, x_{1}^{2 m^{2}+w} x_{2} x_{3}\right\rangle .
$$

Since the Hilbert function of $K\left[x_{1}, x_{2}, x_{3}, x_{4}\right] / I\left(\mathbf{n}^{\prime}\right)_{*}$ is equal to the Hilbert function of $K\left[x_{1}, x_{2}, x_{3}, x_{4}\right] /\left\langle\operatorname{LT}\left(I\left(\mathbf{n}^{\prime}\right)_{*}\right)\right\rangle$, it is sufficient to compute the Hilbert function of the latter. Let

$$
\begin{gathered}
J_{0}=\left\langle\operatorname{LT}\left(I\left(\mathbf{n}^{\prime}\right)_{*}\right)\right\rangle, J_{1}=\left\langle x_{2}^{2}, x_{3}^{2}, x_{4}^{4 m^{2}+2 w+3}, x_{2} x_{4}^{2 m^{2}+w+3}, x_{3} x_{4}^{2 m^{2}+w}\right\rangle, \\
J_{2}=\left\langle x_{2}^{2}, x_{3}^{2}, x_{4}^{4 m^{2}+2 w+3}, x_{2} x_{4}^{2 m^{2}+w+3}\right\rangle, J_{3}=\left\langle x_{2}^{2}, x_{3}^{2}, x_{4}^{4 m^{2}+2 w+3}\right\rangle .
\end{gathered}
$$

Remark that $J_{i}=\left\langle J_{i+1}, q_{i}\right\rangle$, where $q_{0}=x_{1}^{2 m^{2}+w} x_{2} x_{3}, q_{1}=x_{3} x_{4}^{2 m^{2}+w}$ and $q_{2}=$ $x_{2} x_{4}^{2 m^{2}+w+3}$. We apply Proposition 2.11 to the ideal $J_{i}$ for $0 \leq i \leq 2$, so

$$
p\left(J_{i}\right)=p\left(J_{i+1}\right)-t^{\operatorname{deg}\left(q_{i}\right)} p\left(J_{i+1}:\left\langle q_{i}\right\rangle\right) .
$$

Note that $\operatorname{deg}\left(q_{0}\right)=2 m^{2}+w+2, \operatorname{deg}\left(q_{1}\right)=2 m^{2}+w+1$ and $\operatorname{deg}\left(q_{2}\right)=2 m^{2}+$ $w+4$. In this case, it holds that $J_{1}:\left\langle q_{0}\right\rangle=\left\langle x_{2}, x_{3}, x_{4}^{2 m^{2}+w}\right\rangle, J_{2}:\left\langle q_{1}\right\rangle=$ $\left\langle x_{2}^{2}, x_{3}, x_{4}^{2 m^{2}+w+3}, x_{2} x_{4}^{3}\right\rangle$ and $J_{3}:\left\langle q_{2}\right\rangle=\left\langle x_{2}, x_{3}^{2}, x_{4}^{2 m^{2}+w}\right\rangle$. We have that

$$
p\left(J_{3}\right)=(1-t)^{3}\left(1+3 t+4 t^{2}+\cdots+4 t^{4 m^{2}+2 w+2}+3 t^{4 m^{2}+2 w+3}+t^{4 m^{2}+2 w+4}\right) .
$$

Substituting all these recursively in Equation (2.1), we obtain that the Hilbert series of $K\left[x_{1}, x_{2}, x_{3}, x_{4}\right] / J_{0}$ is

$$
\frac{1+3 t+4 t^{2}+\cdots+4 t^{2 m^{2}+w}+3 t^{2 m^{2}+w+1}+t^{2 m^{2}+w+2}+t^{2 m^{2}+w+3}+t^{4 m^{2}+2 w+2}}{1-t} .
$$

Since the numerator does not have any negative coefficients, the Hilbert function of $K\left[x_{1}, x_{2}, x_{3}, x_{4}\right] / J_{0}$ is non-decreasing whenever $\operatorname{gcd}\left(n_{1}^{\prime}, n_{2}^{\prime}, n_{3}^{\prime}, n_{4}^{\prime}\right)=1$. 


\section{The CASE (B)}

In this section we assume that after permuting variables, if necessary, $S=\left\{x_{1}^{a_{1}}-\right.$ $\left.x_{2}^{a_{2}}, x_{3}^{a_{3}}-x_{1}^{u_{1}} x_{2}^{u_{2}}, x_{4}^{a_{4}}-x_{1}^{v_{1}} x_{2}^{v_{2}} x_{3}^{v_{3}}\right\}$ is a minimal generating set of $I(\mathbf{n})$. Proposition 3.1 will be useful in the proof of Theorem 3.2

Proposition 3.1. Let $B=\left\{f_{1}=x_{1}^{b_{1}}-x_{2}^{b_{2}}, f_{2}=x_{3}^{b_{3}}-x_{1}^{c_{1}} x_{2}^{c_{2}}, f_{3}=x_{4}^{b_{4}}-\right.$ $\left.x_{1}^{m_{1}} x_{2}^{m_{2}} x_{3}^{m_{3}}\right\}$ be a set of binomials in $K\left[x_{1}, \ldots, x_{4}\right]$, where $b_{i} \geq 1$ for all $1 \leq i \leq 4$, at least one of $c_{1}, c_{2}$ is non-zero and at least one of $m_{1}, m_{2}$ and $m_{3}$ is non-zero. Let $n_{1}=b_{2} b_{3} b_{4}, n_{2}=b_{1} b_{3} b_{4}, n_{3}=b_{4}\left(b_{1} c_{2}+c_{1} b_{2}\right), n_{4}=m_{3}\left(b_{1} c_{2}+b_{2} c_{1}\right)+b_{3}\left(b_{1} m_{2}+\right.$ $\left.m_{1} b_{2}\right)$. If $\operatorname{gcd}\left(n_{1}, \ldots, n_{4}\right)=1$, then $I(\mathbf{n})$ is a complete intersection ideal generated by the binomials $f_{1}, f_{2}, f_{3}$.

Proof. Consider the vectors $\mathbf{d}_{1}=\left(b_{1},-b_{2}, 0,0\right), \mathbf{d}_{2}=\left(-c_{1},-c_{2}, b_{3}, 0\right)$ and $\mathbf{d}_{3}=$ $\left(-m_{1},-m_{2},-m_{3}, b_{4}\right)$. Clearly $\mathbf{d}_{i} \in \operatorname{ker}_{\mathbb{Z}}\left(n_{1}, \ldots, n_{4}\right)$ for $1 \leq i \leq 3$, so the lattice $L=\sum_{i=1}^{3} \mathbb{Z} \mathbf{d}_{i}$ is a subset of $\operatorname{ker}_{\mathbb{Z}}\left(n_{1}, \ldots, n_{4}\right)$. Let

$$
M=\left(\begin{array}{ccc}
b_{1} & -c_{1} & -m_{1} \\
-b_{2} & -c_{2} & -m_{2} \\
0 & b_{3} & -m_{3} \\
0 & 0 & b_{4}
\end{array}\right)
$$

then the rank of $M$ equals 3 . We will prove that the invariant factors $\delta_{1}, \delta_{2}$ and $\delta_{3}$ of $M$ are all equal to 1 . The greatest common divisor of all non-zero $3 \times 3$ minors of $M$ equals the greatest common divisor of the integers $n_{1}, n_{2}, n_{3}$ and $n_{4}$. But $\operatorname{gcd}\left(n_{1}, \ldots, n_{4}\right)=1$, so $\delta_{1} \delta_{2} \delta_{3}=1$ and therefore $\delta_{1}=\delta_{2}=\delta_{3}=1$. Note that the rank of the lattice $\operatorname{ker}_{\mathbb{Z}}\left(n_{1}, \ldots, n_{4}\right)$ is 3 and also equals the rank of $L$. By [17, Lemma 8.2.5] we have that $L=\operatorname{ker}_{\mathbb{Z}}\left(n_{1}, \ldots, n_{4}\right)$. Now the transpose $M^{t}$ of $M$ is mixed dominating. By [5, Theorem 2.9] the ideal $I(\mathbf{n})$ is a complete intersection on $f_{1}, f_{2}$ and $f_{3}$.

Theorem 3.2. Let $I(\mathbf{n})$ be a complete intersection ideal generated by the binomials $f_{1}=x_{1}^{a_{1}}-x_{2}^{a_{2}}, f_{2}=x_{3}^{a_{3}}-x_{1}^{u_{1}} x_{2}^{u_{2}}$ and $f_{3}=x_{4}^{a_{4}}-x_{1}^{v_{1}} x_{2}^{v_{2}} x_{3}^{v_{3}}$. Then there exist vectors $\mathbf{b}_{i}, 1 \leq i \leq 22$, in $\mathbb{N}^{4}$ such that for all $w>0$, the toric ideal $I\left(\mathbf{n}+w \mathbf{b}_{i}\right)$ is a complete intersection whenever the entries of $\mathbf{n}+w \mathbf{b}_{i}$ are relatively prime.

Proof. By [11, Theorem 6] $n_{1}=a_{2} a_{3} a_{4}, n_{2}=a_{1} a_{3} a_{4}, n_{3}=a_{4}\left(a_{1} u_{2}+u_{1} a_{2}\right)$, $n_{4}=v_{3}\left(a_{1} u_{2}+a_{2} u_{1}\right)+a_{3}\left(a_{1} v_{2}+v_{1} a_{2}\right)$. Let $\mathbf{b}_{1}=\left(a_{2} a_{3}, a_{1} a_{3}, a_{1} u_{2}+u_{1} a_{2}, a_{2} a_{3}\right)$ and consider the set $B=\left\{f_{1}, f_{2}, f_{4}=x_{4}^{a_{4}+w}-x_{1}^{v_{1}+w} x_{2}^{v_{2}} x_{3}^{v_{3}}\right\}$. Then $n_{1}+w a_{2} a_{3}=$ $a_{2} a_{3}\left(a_{4}+w\right), n_{2}+w a_{1} a_{3}=a_{1} a_{3}\left(a_{4}+w\right), n_{3}+w\left(a_{1} u_{2}+u_{1} a_{2}\right)=\left(a_{4}+w\right)\left(a_{1} u_{2}+u_{1} a_{2}\right)$ and $n_{4}+w a_{2} a_{3}=v_{3}\left(a_{1} u_{2}+a_{2} u_{1}\right)+a_{3}\left(a_{1} v_{2}+\left(v_{1}+w\right) a_{2}\right)$. By Proposition 3.1 for every $w>0$, the ideal $I\left(\mathbf{n}+w \mathbf{b}_{1}\right)$ is a complete intersection on $f_{1}, f_{2}$ and $f_{4}$ whenever the entries of $\mathbf{n}+w \mathbf{b}_{1}$ are relatively prime. Consider the vectors $\mathbf{b}_{2}=\left(a_{2} a_{3}, a_{1} a_{3}, a_{1} u_{2}+u_{1} a_{2}, a_{1} a_{3}\right), \mathbf{b}_{3}=\left(a_{2} a_{3}, a_{1} a_{3}, a_{1} u_{2}+u_{1} a_{2}, a_{1} u_{2}+u_{1} a_{2}\right)$, $\mathbf{b}_{4}=\left(0,0,0, a_{3}\left(a_{1}+a_{2}\right)\right), \mathbf{b}_{5}=\left(0,0,0, a_{1} u_{2}+a_{2} u_{1}+a_{2} a_{3}\right)$ and $\mathbf{b}_{6}=\left(0,0,0, a_{1} u_{2}+\right.$ $\left.a_{2} u_{1}+a_{1} a_{3}\right)$. By Proposition 3.1 for every $w>0, I\left(\mathbf{n}+w \mathbf{b}_{2}\right)$ is a complete intersection on $f_{1}, f_{2}$ and $x_{4}^{a_{4}+w}-x_{1}^{v_{1}} x_{2}^{v_{2}+w} x_{3}^{v_{3}}$ whenever the entries of $\mathbf{n}+w \mathbf{b}_{2}$ are relatively prime, $I\left(\mathbf{n}+w \mathbf{b}_{3}\right)$ is a complete intersection on $f_{1}, f_{2}$ and $x_{4}^{a_{4}+w}-$ $x_{1}^{v_{1}} x_{2}^{v_{2}} x_{3}^{v_{3}+w}$ whenever the entries of $\mathbf{n}+w \mathbf{b}_{3}$ are relatively prime, and $I\left(\mathbf{n}+w \mathbf{b}_{4}\right)$ is a complete intersection on $f_{1}, f_{2}$ and $x_{4}^{a_{4}}-x_{1}^{v_{1}+w} x_{2}^{v_{2}+w} x_{3}^{v_{3}}$ whenever the entries of $\mathbf{n}+w \mathbf{b}_{4}$ are relatively prime. Furthermore for every $w>0, I\left(\mathbf{n}+w \mathbf{b}_{5}\right)$ is a complete intersection on $f_{1}, f_{2}$ and $x_{4}^{a_{4}}-x_{1}^{v_{1}+w} x_{2}^{v_{2}} x_{3}^{v_{3}+w}$ whenever the entries of $\mathbf{n}+w \mathbf{b}_{5}$ are relatively prime, and $I\left(\mathbf{n}+w \mathbf{b}_{6}\right)$ is a complete intersection on $f_{1}, f_{2}$ and $x_{4}^{a_{4}}-x_{1}^{v_{1}} x_{2}^{v_{2}+w} x_{3}^{v_{3}+w}$ whenever the entries of $\mathbf{n}+w \mathbf{b}_{6}$ are relatively prime. Consider the vectors $\mathbf{b}_{7}=\left(a_{2} a_{3}, a_{1} a_{3}, a_{1} u_{2}+u_{1} a_{2}, a_{3}\left(a_{1}+a_{2}\right)\right), \mathbf{b}_{8}=$ $\left(a_{2} a_{3}, a_{1} a_{3}, a_{1} u_{2}+u_{1} a_{2}, a_{1} u_{2}+u_{1} a_{2}+a_{2} a_{3}\right), \mathbf{b}_{9}=\left(a_{2} a_{3}, a_{1} a_{3}, a_{1} u_{2}+u_{1} a_{2}, a_{1} u_{2}+\right.$ 
$\left.u_{1} a_{2}+a_{1} a_{3}\right), \mathbf{b}_{10}=\left(0,0,0, a_{1} u_{2}+a_{2} u_{1}+a_{3}\left(a_{1}+a_{2}\right)\right), \mathbf{b}_{11}=\left(a_{2} a_{3}, a_{1} a_{3}, a_{1} u_{2}+\right.$ $\left.u_{1} a_{2}, 0\right), \mathbf{b}_{12}=\left(0,0,0, a_{2} a_{3}\right), \mathbf{b}_{13}=\left(0,0,0, a_{1} a_{3}\right), \mathbf{b}_{14}=\left(0,0,0, a_{1} u_{2}+a_{2} u_{1}\right)$ and $\mathbf{b}_{15}=\left(a_{2} a_{3}, a_{1} a_{3}, a_{1} u_{2}+u_{1} a_{2}, a_{1} u_{2}+u_{1} a_{2}+a_{3}\left(a_{1}+a_{2}\right)\right)$. Using Proposition $3.1 \mathrm{we}$ have that for all $w>0$, the ideal $I\left(\mathbf{n}+w \mathbf{b}_{i}\right), 7 \leq i \leq 15$, is a complete intersection whenever the entries of $\mathbf{n}+w \mathbf{b}_{i}$ are relatively prime. Finally consider the vectors $\mathbf{b}_{16}=\left(a_{3} a_{4}, a_{3} a_{4}, a_{4}\left(u_{1}+u_{2}\right), v_{3}\left(u_{1}+u_{2}\right)+a_{3}\left(v_{1}+v_{2}\right)\right), \mathbf{b}_{17}=\left(0, a_{3} a_{4}, a_{4} u_{2}, u_{2} v_{3}+\right.$ $\left.a_{3} v_{2}\right), \mathbf{b}_{18}=\left(a_{3} a_{4}, 0, a_{4} u_{1}, u_{1} v_{3}+v_{1} a_{3}\right), \mathbf{b}_{19}=\left(a_{2} a_{4}, a_{1} a_{4}, a_{2} a_{4}, a_{2} v_{3}+a_{1} v_{2}+v_{1} a_{2}\right)$, $\mathbf{b}_{20}=\left(a_{2} a_{4}, a_{1} a_{4}, a_{1} a_{4}, a_{1} v_{3}+a_{1} v_{2}+v_{1} a_{2}\right), \mathbf{b}_{21}=\left(a_{2} a_{4}, a_{1} a_{4}, a_{4}\left(a_{1}+a_{2}\right), v_{3}\left(a_{1}+\right.\right.$ $\left.\left.a_{2}\right)+a_{1} v_{2}+v_{1} a_{2}\right)$ and $\mathbf{b}_{22}=\left(0,0, a_{4}\left(a_{1}+a_{2}\right), v_{3}\left(a_{1}+a_{2}\right)+a_{3}\left(a_{1}+a_{2}\right)\right)$. It is easy to see that for all $w>0$, the ideal $I\left(\mathbf{n}+w \mathbf{b}_{i}\right), 16 \leq i \leq 22$, is a complete intersection whenever the entries of $\mathbf{n}+w \mathbf{b}_{i}$ are relatively prime. For instance $I\left(\mathbf{n}+w \mathbf{b}_{22}\right)$ is a complete intersection on the binomials $f_{1}, x_{3}^{a_{3}}-x_{1}^{u_{1}+w} x_{2}^{u_{2}+w}$ and $x_{4}^{a_{4}}-x_{1}^{v_{1}+w} x_{2}^{v_{2}+w} x_{3}^{v_{3}}$.

Example 3.3. Let $\mathbf{n}=(231,770,1023,674)$, then $I(\mathbf{n})$ is a complete intersection on the binomials $x_{1}^{10}-x_{2}^{3}, x_{3}^{7}-x_{1}^{11} x_{2}^{6}$ and $x_{4}^{11}-x_{1} x_{2}^{8} x_{3}$. Here $a_{1}=10, a_{2}=3$, $a_{3}=7, a_{4}=11, u_{1}=11, u_{2}=6, v_{1}=1, v_{2}=8$ and $v_{3}=1$. Consider the vector $\mathbf{b}_{22}=(0,0,143,104)$, then for all $w \geq 0$ the ideal $I\left(\mathbf{n}+w \mathbf{b}_{22}\right)$ is a complete intersection on $x_{1}^{10}-x_{2}^{3}, x_{3}^{7}-x_{1}^{11+w} x_{2}^{6+w}$ and $x_{4}^{11}-x_{1}^{1+w} x_{2}^{8+w} x_{3}$ whenever $\operatorname{gcd}(231,770,1023+143 w, 674+104 w)=1$. In fact, $I\left(\mathbf{n}+w \mathbf{b}_{22}\right)$ is minimally generated by $x_{1}^{10}-x_{2}^{3}, x_{3}^{7}-x_{1}^{11+w} x_{2}^{6+w}$ and $x_{4}^{11}-x_{1}^{11+w} x_{2}^{5+w} x_{3}$. Remark that $231=$ $\min \{231,770,1023+143 w, 674+104 w\}$. The set $\left\{x_{1}^{10}-x_{2}^{3}, x_{3}^{7}-x_{1}^{11+w} x_{2}^{6+w}, x_{4}^{11}-\right.$ $\left.x_{1}^{11+w} x_{2}^{5+w} x_{3}\right\}$ is a standard basis for $I\left(\mathbf{n}+w \mathbf{b}_{22}\right)$ with respect to the negative degree reverse lexicographical order with $x_{4}>x_{3}>x_{2}>x_{1}$. So $I\left(\mathbf{n}+w \mathbf{b}_{22}\right)_{*}$ is a complete intersection on $x_{2}^{3}, x_{3}^{7}$ and $x_{4}^{11}$, and therefore for every $w \geq 0$ the monomial curve $C\left(\mathbf{n}+w \mathbf{b}_{22}\right)$ has Cohen-Macaulay tangent cone at the origin whenever $\operatorname{gcd}(231,770,1023+143 w, 674+104 w)=1$. Let $\mathbf{b}_{16}=(77,77,187,80)$. For every $w \geq 0, I\left(\mathbf{n}+w \mathbf{b}_{16}\right)$ is a complete intersection on $x_{1}^{10+w}-x_{2}^{3+w}, x_{3}^{7}-x_{1}^{11} x_{2}^{6}$ and $x_{4}^{11}-x_{1} x_{2}^{8} x_{3}$ whenever $\operatorname{gcd}(231+77 w, 770+77 w, 1023+187 w, 674+80 w)=1$. Note that $231+77 w=\min \{231+77 w, 770+77 w, 1023+187 w, 674+80 w\}$. For $0 \leq w \leq 5$ the set $\left\{x_{1}^{10+w}-x_{2}^{3+w}, x_{3}^{7}-x_{1}^{11} x_{2}^{6}, x_{4}^{11}-x_{1}^{11+w} x_{2}^{5-w} x_{3}\right\}$ is a standard basis for $I\left(\mathbf{n}+w \mathbf{b}_{16}\right)$ with respect to the negative degree reverse lexicographical order with $x_{4}>x_{3}>x_{2}>x_{1}$. Thus $I\left(\mathbf{n}+w \mathbf{b}_{16}\right)_{*}$ is minimally generated by $\left\{x_{2}^{3+w}, x_{3}^{7}, x_{4}^{11}\right\}$, so for $0 \leq w \leq 5$ the monomial curve $C\left(\mathbf{n}+w \mathbf{b}_{16}\right)$ has Cohen-Macaulay tangent cone at the origin whenever $\operatorname{gcd}(231+77 w, 770+77 w, 1023+187 w, 674+80 w)=1$. Suppose that there is $w \geq 6$ such that $C\left(\mathbf{n}+w \mathbf{b}_{16}\right)$ has Cohen-Macaulay tangent cone at the origin. Then $x_{2}^{8} x_{3} \in I\left(\mathbf{n}+w \mathbf{b}_{16}\right)_{*}:\left\langle x_{1}\right\rangle$ and therefore $x_{2}^{8} x_{3} \in I\left(\mathbf{n}+w \mathbf{b}_{16}\right)_{*}$. Thus $x_{2}^{8} x_{3}$ is divided by $x_{2}^{3+w}$, a contradiction. Consequently for every $w \geq 6$ the monomial curve $C\left(\mathbf{n}+w \mathbf{b}_{16}\right)$ does not have Cohen-Macaulay tangent cone at the origin whenever $\operatorname{gcd}(231+77 w, 770+77 w, 1023+187 w, 674+80 w)=1$.

Theorem 3.4. Let $I(\mathbf{n})$ be a complete intersection ideal generated by the binomials $f_{1}=x_{1}^{a_{1}}-x_{2}^{a_{2}}, f_{2}=x_{3}^{a_{3}}-x_{1}^{u_{1}} x_{2}^{u_{2}}$ and $f_{3}=x_{4}^{a_{4}}-x_{1}^{v_{1}} x_{2}^{v_{2}} x_{3}^{v_{3}}$. Consider the vector $\mathbf{d}=\left(0,0, a_{4}\left(a_{1}+a_{2}\right), v_{3}\left(a_{1}+a_{2}\right)+a_{3}\left(a_{1}+a_{2}\right)\right)$. Then there exists a non-negative integer $w_{1}$ such that for all $w \geq w_{1}$, the ideal $I(\mathbf{n}+w \mathbf{d})_{*}$ is a complete intersection whenever the entries of $\mathbf{n}+w \mathbf{d}$ are relatively prime.

Proof. By Theorem 3.2 for all $w \geq 0$, the ideal $I(\mathbf{n}+w \mathbf{d})$ is minimally generated by $G=\left\{f_{1}, f_{4}=x_{3}^{a_{3}}-x_{1}^{u_{1}+w} x_{2}^{u_{2}+w}, f_{5}=x_{4}^{a_{4}}-x_{1}^{v_{1}+w} x_{2}^{v_{2}+w} x_{3}^{v_{3}}\right\}$ whenever the entries of $\mathbf{n}+w \mathbf{d}$ are relatively prime. Let $w_{1}$ be the smallest non-negative integer greater than or equal to $\max \left\{\frac{a_{3}-u_{1}-u_{2}}{2}, \frac{a_{4}-v_{1}-v_{2}-v_{3}}{2}\right\}$. Then $a_{3} \leq u_{1}+u_{2}+2 w_{1}$ and $a_{4} \leq v_{1}+v_{2}+v_{3}+2 w_{1}$. It is easy to prove that for every $w \geq w_{1}$ the set $G$ is a standard basis for $I(\mathbf{n}+w \mathbf{d})$ with respect to the negative degree reverse lexicographical order with $x_{4}>x_{3}>x_{2}>x_{1}$. Note that $\operatorname{LM}\left(f_{1}\right)$ is either $x_{1}^{a_{1}}$ 
or $x_{2}^{a_{2}}, \operatorname{LM}\left(f_{4}\right)=x_{3}^{a_{3}}$ and $\operatorname{LM}\left(f_{5}\right)=x_{4}^{a_{4}}$. By [8, Lemma 5.5.11] $I(\mathbf{n}+w \mathbf{d})_{*}$ is generated by the least homogeneous summands of the elements in the standard basis $G$. Thus for all $w \geq w_{1}$, the ideal $I(\mathbf{n}+w \mathbf{d})_{*}$ is a complete intersection whenever the entries of $\mathbf{n}+w \mathbf{d}$ are relatively prime.

Proposition 3.5. Let $I(\mathbf{n})$ be a complete intersection ideal generated by the binomials $f_{1}=x_{1}^{a_{1}}-x_{2}^{a_{2}}, f_{2}=x_{3}^{a_{3}}-x_{1}^{u_{1}} x_{2}^{u_{2}}$ and $f_{3}=x_{4}^{a_{4}}-x_{1}^{v_{1}} x_{2}^{v_{2}}$, where $v_{1}>0$ and $v_{2}>0$. Assume that $a_{2}<a_{1}, a_{3}<u_{1}+u_{2}, v_{2}<a_{2}$ and $a_{1}+v_{1} \leq a_{2}-v_{2}+a_{4}$. Then there exists a vector $\mathbf{b}$ in $\mathbb{N}^{4}$ such that for all $w \geq 0$, the ideal $I(\mathbf{n}+w \mathbf{b})_{*}$ is almost complete intersection whenever the entries of $\mathbf{n}+w \mathbf{b}$ are relatively prime.

Proof. From the assumptions we deduce that $v_{1}+v_{2}<a_{4}$. Consider the vector $\mathbf{b}=\left(a_{2} a_{3}, a_{1} a_{3}, a_{1} u_{2}+u_{1} a_{2}, a_{2} a_{3}\right)$. For every $w \geq 0$ the ideal $I(\mathbf{n}+w \mathbf{b})$ is a complete intersection on $f_{1}, f_{2}$ and $f_{4}=x_{4}^{a_{4}+w}-x_{1}^{v_{1}+w} x_{2}^{v_{2}}$ whenever the entries of $\mathbf{n}+w \mathbf{b}$ are relatively prime. We claim that the set $G=\left\{f_{1}, f_{2}, f_{4}, f_{5}=\right.$ $\left.x_{1}^{a_{1}+v_{1}+w}-x_{2}^{a_{2}-v_{2}} x_{4}^{a_{4}+w}\right\}$ is a standard basis for $I(\mathbf{n}+w \mathbf{b})$ with respect to the negative degree reverse lexicographical order with $x_{3}>x_{2}>x_{1}>x_{4}$. Note that $\operatorname{LM}\left(f_{1}\right)=x_{2}^{a_{2}}, \operatorname{LM}\left(f_{2}\right)=x_{3}^{a_{3}}, \operatorname{LM}\left(f_{4}\right)=x_{1}^{v_{1}+w} x_{2}^{v_{2}}$ and $\operatorname{LM}\left(f_{5}\right)=x_{1}^{a_{1}+v_{1}+w}$. Also $\operatorname{spoly}\left(f_{1}, f_{4}\right)=-f_{5}$. It suffices to show that $\operatorname{NF}\left(\operatorname{spoly}\left(f_{4}, f_{5}\right) \mid G\right)=0$. We compute $\operatorname{spoly}\left(f_{4}, f_{5}\right)=x_{2}^{a_{2}} x_{4}^{a_{4}+w}-x_{1}^{a_{1}} x_{4}^{a_{4}+w}$. Then $\operatorname{LM}\left(\operatorname{spoly}\left(f_{4}, f_{5}\right)\right)=x_{2}^{a_{2}} x_{4}^{a_{4}+w}$ and only $\operatorname{LM}\left(f_{1}\right)$ divides $\operatorname{LM}\left(\operatorname{spoly}\left(f_{4}, f_{5}\right)\right)$. Moreover ecart $\left(\operatorname{spoly}\left(f_{4}, f_{5}\right)\right)=a_{1}-a_{2}=$ $\operatorname{ecart}\left(f_{1}\right)$. So $\operatorname{spoly}\left(f_{1}, \operatorname{spoly}\left(f_{4}, f_{5}\right)\right)=0$ and also $\operatorname{NF}\left(\operatorname{spoly}\left(f_{4}, f_{5}\right) \mid G\right)=0$. Thus

(1) If $a_{1}+v_{1}<a_{2}-v_{2}+a_{4}$, then $I(\mathbf{n}+w \mathbf{b})_{*}$ is minimally generated by $\left\{x_{2}^{a_{2}}, x_{3}^{a_{3}}, x_{1}^{v_{1}+w} x_{2}^{v_{2}}, x_{1}^{a_{1}+v_{1}+w}\right\}$.

(2) If $a_{1}+v_{1}=a_{2}-v_{2}+a_{4}$, then $I(\mathbf{n}+w \mathbf{b})_{*}$ is minimally generated by $\left\{x_{2}^{a_{2}}, x_{3}^{a_{3}}, x_{1}^{v_{1}+w} x_{2}^{v_{2}}, f_{5}\right\}$.

\section{REFERENCES}

[1] F. Arslan, P. Mete, M. Sahin, Gluing and Hilbert functions of monomial curves, Proc. Amer. Math. Soc. 137 (2009) 2225-2232.

[2] D. Bayer, M. Stillman, Computation of Hilbert functions, J. Symbolic Comput. 14 (1992) 31-50.

[3] CoCoATeam, CoCoA: A system for doing computations in commutative algebra, available at http://cocoa.dima.unige.it

[4] R. Conaway, F. Gotti, J. Horton, C. O'Neill, R. Pelayo, M. Pracht, B. Wissman, Minimal presentations of shifted numerical monoids, Internat. J. Algebra Comput. 28 (2018) 53-68.

[5] K. Fischer, J. Shapiro, Mixed matrices and binomial ideals, J. Pure Appl. Algebra 113 (1996) $39-54$.

[6] A. Garcia, Cohen-Macaulayness of the associated graded of a semigroup ring, Comm. Algebra 10 (1982) 393-415.

[7] P. Gimenez, H. Srinivasan, A note on Gorenstein monomial curves, Bull. Braz. Math. Soc. (N.S.) 4 (2014) 671-678.

[8] G.-M. Greuel, G. Pfister, A Singular Introduction to Commutative Algebra, Springer-Verlag, 2002.

[9] N. Jacobson, Basic Algebra I, Second edition, W. H. Freeman and Company, New York, 1985.

[10] A. Katsabekis, I. Ojeda, An indispensable classification of monomial curves in $\mathbb{A}^{4}(K)$, Pacific J. Math. 268 (2014) 95-116.

[11] J. Kraft, Singularity of monomial curves in $A^{3}$ and Gorenstein monomial curves in $A^{4}$, Canad. J. Math. 37 (1985) 872-892.

[12] A. Oneto, F. Strazzanti, G. Tamone, One-dimensional Gorenstein local rings with decreasing Hilbert function, J. Algebra 489 (2017) 91-114

[13] M. Rossi, Hilbert functions of Cohen-Macaulay local rings, Commutative Algebra and its Connections to Geometry, Contemporary Math 555 (2011), AMS, 173-200.

[14] T. Shibuta, Cohen-Macaulayness of almost complete intersection tangent cones, J. Algebra 319 (2008) 3222-3243.

[15] D.I. Stamate, Betti numbers for numerical semigroup rings, in: Multigraded Algebra and Applications-NSA 24, 2016, Springer Proceedings in Mathematics and Statistics, 238 (eds. V. Ene and E. Miller) (Springer, Cham, 2018). 
[16] B. Sturmfels, Gröbner Bases and Convex Polytopes, University Lecture Series, No. 8, American Mathematical Society Providence, RI 1995.

[17] R.H. Villarreal, Monomial Algebras, Second Edition, Monographs and Research Notes in Mathematics, CRC Press, Boca Raton, FL, 2015.

[18] T. Vu, Periodicity of Betti numbers of monomial curves, J. Algebra 418 (2014) 66-90.

Department of Mathematics, Bilkent University, 06800 Ankara, Turkey

E-mail address: katsampekis@bilkent.edu.tr 